OPEN ACCESS

Edited by:

Juan Zhou,

Duke-NUS Medical School,

Singapore

Reviewed by:

Dong-Hoon Lee,

University of Sydney, Australia

Claudia Altamura

Università Campus Bio-Medico, Italy

*Correspondence:

Junrong $L i$

liry612@163.com

Received: 14 August 2018 Accepted: 09 November 2018 Published: 28 November 2018

Citation:

Chen X, Chen X, Chen Y, Xu M, Yu T and $\mathrm{Li} J$ (2018) The Impact of

Intracerebral Hemorrhage on the

Progression of White Matter

Hyperintensity.

Front. Hum. Neurosci. 12:471.

doi: 10.3389/fnhum.2018.00471

\section{The Impact of Intracerebral Hemorrhage on the Progression of White Matter Hyperintensity}

\author{
Xuemei Chen ${ }^{1}$, Xin Chen ${ }^{2}$, Yan Chen ${ }^{2}$, Manman $X u^{2}$, Tingting $Y u^{2}$ and Junrong $L i^{1 *}$ \\ ${ }^{1}$ Department of Neurology, The Affiliated Jiangning Hospital of Nanjing Medical University, Nanjing, China, ${ }^{2}$ Department \\ of Neurology, The Affiliated Drum Tower Hospital of Nanjing Medical University, Nanjing, China
}

Objective: The exact relationship between white matter hyperintensity $(\mathrm{WMH})$ and intracerebral hemorrhage $(\mathrm{ICH})$ after $\mathrm{ICH}$ remains unclear. In this retrospective study, we investigated whether patients with $\mathrm{ICH}$ had more severe WMH progression.

Patients and Methods: A total of 2,951 patients aged $\geq 40$ years with $\mathrm{ICH}$ who received brain computed tomography (CT) imaging within $12 \mathrm{~h}$ of $\mathrm{ICH}$ symptom onset were screened. Ninety patients with two fluid-attenuated inversion recovery (FLAIR) magnetic resonance imaging (MRI) assessments, including 36 patients with Lobar $\mathrm{ICH}, 40$ with basal ganglia region $\mathrm{ICH}$ and 14 with $\mathrm{ICH}$ at other sites, were included in the final study. We selected 90 age- and gender-matched healthy individuals with two MRI scans as the control group. The WMH volumes at baseline and follow-up were assessed using the FLAIR image by MRICRON and ITK-SNAP software, while the hematoma volumes were calculated based on the CT images using ITK-SNAP software.

Results: The annual progression rate of $\mathrm{WMH}$ was significantly higher in the $\mathrm{ICH}$ group compared with the control group $(p<0.05)$. Furthermore, $\mathrm{WMH}$ progression was associated with the $\mathrm{ICH}$ volume. The largest $\mathrm{ICH}$ volume $(>30 \mathrm{~mL}$ ) was associated with the highest annual progression rate of $\mathrm{WMH}(0<0.05)$. In contrast, no trend toward an association between $\mathrm{ICH}$ location and the annual progression rate of $\mathrm{WMH}$ was observed $(p>0.05)$.

Conclusions: Our results showed that $\mathrm{ICH}$ patients had more severe $\mathrm{WMH}$ progression and that larger $\mathrm{ICH}$ volume was related to greater progression of $\mathrm{WMH}$ after $\mathrm{ICH}$. These results could provide important prognostic information about patients with $\mathrm{ICH}$.

Keywords: intracerebral hemorrhage, white matter injury, hematoma, white matter hyperintensity, quantitative analysis

Abbreviations: apoA I, apolipoprotein A-I; BUN, blood urea nitrogen; Cr, creatinine; CRP, C-reactive protein; CSF, cerebrospinal fluid; CT, computed tomography; DBPs, diastolic blood pressures; DTI, diffusion tensor imaging; FBG, fasting blood glucose; FLAIR, fluid-attenuated inversion recovery; GM, gray matter; HbAIc, glycosylated hemoglobin; HDL, high-density lipoprotein; ICH, intracerebral hemorrhage; IVT, intravenous thrombolysis; LDL, low-density lipoprotein; MBP, myelin basic protein; MRI, magnetic resonance imaging; SBPs, systolic blood pressures; TC, total cholesterol; TG, triglycerides; TIA, transient ischemic attack; UA, uric acid; WM, white matter; WMH, white matter hyperintensity; WMI, white matter injury. 


\section{INTRODUCTION}

Intracerebral hemorrhage (ICH) accounts for approximately $10 \%-15 \%$ of all strokes in Western countries and 20\%-30\% of strokes in Asia and has a high mortality and poor functional outcome (Wen et al., 2018). ICH causes gray matter (GM) destruction as well as proximal or distal white matter injury (WMI) due to complex pathophysiological mechanisms. WM fibers, especially those located within the capsula interna, are one of the most vulnerable tissues in hypertensive ICH. WM hyperintensity (WMH) is a neuroimaging finding of WMI characterized by bilateral, mostly symmetrical, hyperintensities on T2-weighted magnetic resonance imaging (MRI; Wardlaw et al., 2013). Approximately $70 \%$ of people over 65 years of age with ICH present with varying degrees of WMH on MRI (de Leeuw et al., 2001; Smith et al., 2017). Increasing WMH volumes are clinically important because they confer risk for developing cognitive impairment, dementia, gait disturbances and depression (Srikanth et al., 2010; Callisaya et al., 2015). Therefore, more attention should be paid to the progression of WMH after ICH.

Previous research has suggested that WMI might reflect the vulnerability of individual brains to pathologic insults and that WMH should be considered when assessing immediate, early, and long-term outcomes after ICH (Zuo et al., 2017). Several examples from the related literature have demonstrated that severe $\mathrm{WMH}$ had an impact on larger ICH volumes and hematoma growth; moreover, progression of WMH was an independent predictor of worse functional outcomes in patients with ICH (Lou et al., 2010; Caprio et al., 2013). Our recent research has also shown that greater severity and progression of WMH resulted in larger ICH volumes at baseline (Chen et al., 2018). Therefore, the progression of WMH is an important factor in determining the prognosis of patients with ICH. However, the current studies about WMI and repair after hypertensive ICH are still rare and scattered (Zuo et al., 2017). In recent years, some animal researches have shown that scores of therapeutic agents and methods were effective in the treatment of WMI after hypertensive $\mathrm{ICH}$, although these drugs and treatment methods were rarely used in clinical practice because of the absence of enough clinical studies to support their use (Zuo et al., 2017). A better understanding of the characteristics of WMH following ICH is essential and may shed new light on treatment options. Therefore, in this retrospective study, we aimed to figure out the impact of ICH on the progression of WMH.

\section{PATIENTS AND METHODS}

\section{Study Population}

A total of 2,951 patients with ICH were studied retrospectively from January 1, 2012 to November 1, 2017, using data from the Jiangning Hospital imaging center of Nanjing Medical University. The inclusion criteria were as follows: all patients (1) were $\geq 40$ years of age; (2) underwent brain computed tomography (CT) within $12 \mathrm{~h}$ of ICH symptom onset;
(3) received fluid-attenuated inversion recovery (FLAIR) MRI (the first MRI was performed within 2 days after onset to exclude other disorders of the nervous system and the second MRI was performed at return visit; the interval time between the two scans was $268.36 \pm 98.54$ days [mean \pm stand deviation (SD)], range from 3 months to 3 years); and (4) had baseline clinical and demographic information available, including demographic characteristics, medical history, physical examination results and laboratory examination findings. The exclusion criteria included having an underlying aneurysm, vascular malformation or tumor, head trauma, venous infarction, Moyamoya disease, hemorrhagic transformation of ischemic infarction and previous surgical evacuation or craniectomy. We selected 90 age- and gendermatched healthy individuals who underwent regular health check at Jiangning Hospital with two MRI scans as the control group (the interval time between the two scans was 300.47.36 \pm 96.06 days [mean $\pm \mathrm{SD}$ ], range from 3 months to 3 years). The exclusion criteria for healthy individuals were as follows: (1) parenchymal hemorrhage or subarachnoid hemorrhage; (2) history of ischemic stroke or cardiogenic cerebral infarction; (3) other neurological diseases (e.g., Parkinson's disease, epilepsy, AD, multiple sclerosis and neuromyelitis optica); (4) systemic disease (cancer, shock, anemia, or thyroid dysfunction); (5) psychiatric disease; and (6) MRI technical difficulties (Figure 1).

The Ethics Committee of Jiangning Hospital, affiliated with Nanjing Medical University, approved this study. The study procedures were conducted according to institutional guidelines.

\section{Risk Factors}

To assess relevant risk factors at baseline, we collected data on patients' clinical and demographic characteristics, including age and sex; comorbid conditions, including history of hypertension, history of diabetes mellitus, presence of coronary heart disease, history of dyslipidemia and history of stroke or transient ischemic attack (TIA); medications used on admission, such as antiplatelet agents, anticoagulants and statins; systolic and diastolic blood pressure (SBP and DBP) on initial evaluation; past or current cigarette or alcohol use; and laboratory results, including tests for glucose [fasting blood glucose (FBG) and glycosylated hemoglobin (HbAIc)], total cholesterol (TC), high-density lipoprotein (HDL), triglycerides (TG), low-density lipoprotein (LDL), Apolipoprotein A-I (apoA I), C-reactive protein $(\mathrm{CRP})$, blood urea nitrogen $(\mathrm{BUN})$, creatinine $(\mathrm{Cr})$ and uric acid (UA).

\section{Radiologic Data}

\section{CT Acquisition and Analysis}

CT scanning was performed on a Lightspeed scanner (GE Medical system) and images were obtained with the following parameters: acquisition matrix $=512 \times 512$, thickness $=10.0 \mathrm{~mm}$. At baseline, the hematoma volumes of the $90 \mathrm{ICH}$ patients were measured with ITK-SNAP software (University of Pennsylvania, Philadelphia, PA, USA) ${ }^{1}$. The

\footnotetext{
${ }_{1 \text { www.itksnap.org }}$
} 


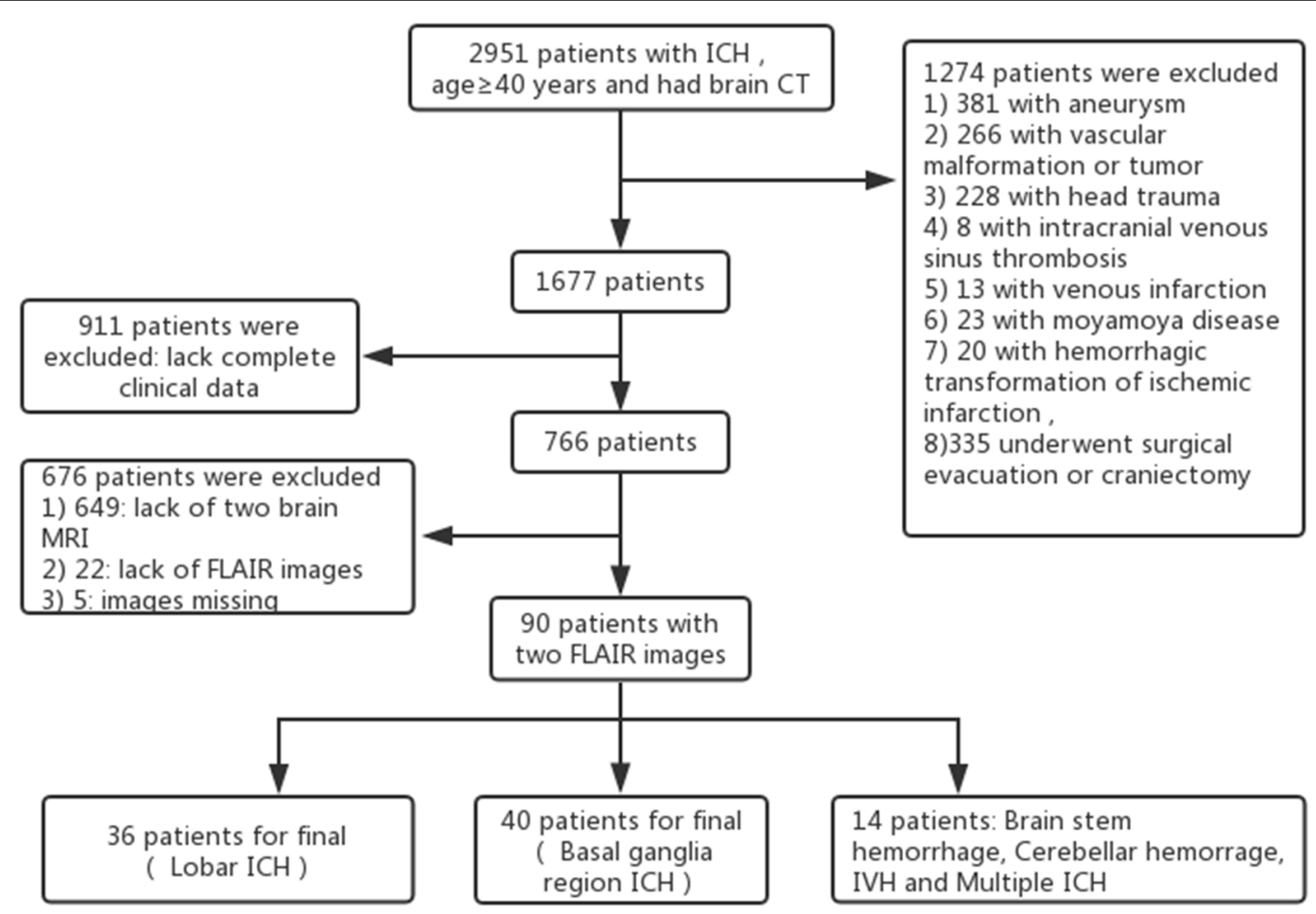

FIGURE 1 | Flowchart of the screening process.

baseline scan was limited to $12 \mathrm{~h}$ because most of the hematoma expansion occurred within the first $24 \mathrm{~h}$ following $\mathrm{ICH}$ onset (Figure 2).

\section{MRI Acquisition and Analysis}

MRI scanning was performed on a 3.0-T scanner (Achieva 3.0 T Ingenia; Philips Medical Systems, Eindhoven, Netherlands) with a 32-channel head coil. High-resolution T1-weighted axial images covering the whole brain were obtained by a 3D-magnetization prepared rapid gradient-echo sequence:
$\mathrm{TR}=9.8 \mathrm{~ms} ; \mathrm{FA}=8^{\circ} ; \mathrm{TE}=4.6 \mathrm{~ms} ; \mathrm{FOV}=256 \times 256 \mathrm{~mm} ;$ acquisition matrix $=256 \times 256$; gap $=0 \mathrm{~mm}$, thickness $=1.0 \mathrm{~mm}$; number of slices $=192$. Additionally, the T2 FLAIR axial images were obtained with the following parameters: $\mathrm{TR}=4,500 \mathrm{~ms}$; $\mathrm{TE}=344 \mathrm{~ms} ; \mathrm{FA}=90^{\circ}$; acquisition matrix $=272 \times 272$; thickness $=1 \mathrm{~mm}$; gap $=0 \mathrm{~mm}$, number of slices $=200$.

\section{Radiological Diagnosis of WMH}

$\mathrm{WMH}$ is defined as hyperintense on T2-weighted or FLAIR sequences but can appear as isointense or hypointense
A

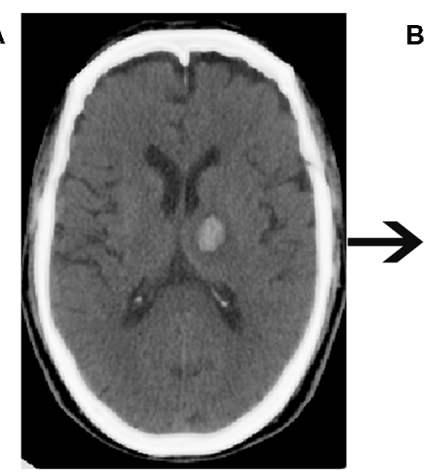

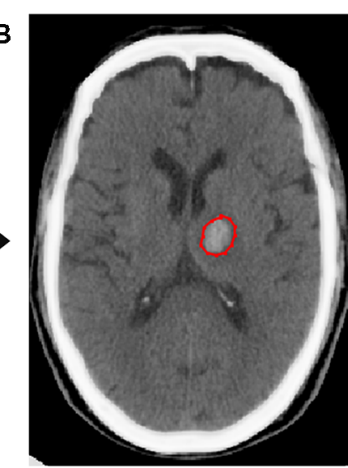

C

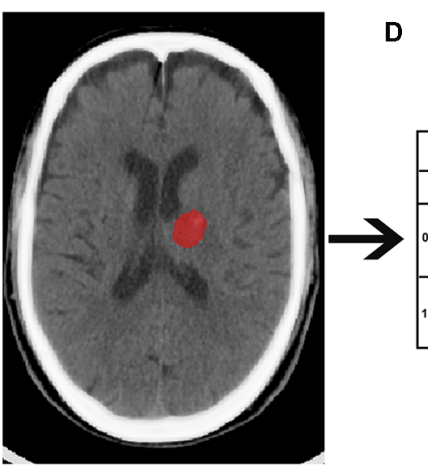

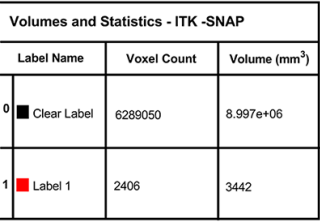

FIGURE 2 | Quantitative measurement of intracerebral hemorrhage (ICH) volume. (A) Original computed tomography (CT) image. (B) The high-density area. (C) A sketch of the high-density area. (D) Calculation of the $\mathrm{ICH}$ volume. 

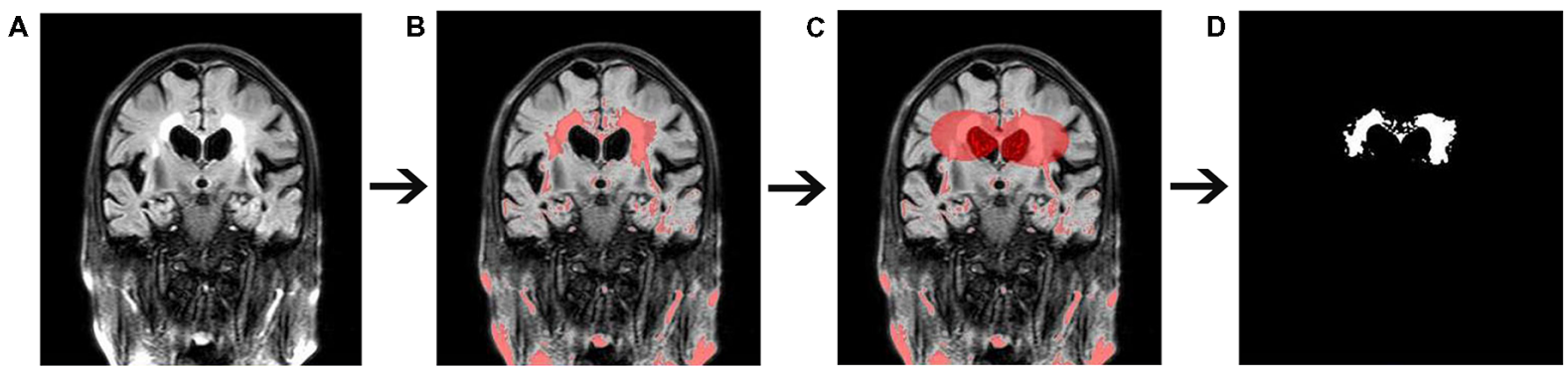

FIGURE 3 | Quantitative measurement of white matter hyperintensity (WMH) volume. (A) Original fluid-attenuated inversion recovery (FLAIR) image. (B) The high-signal area. (C) A sketch of the affected WMH area. (D) Extraction of the affected WMH area.

(although less hypointense than cerebrospinal fluid, CSF) on T1-weighted sequences, depending on the sequence parameters and the severity of the pathological changes. WM lesions, characterized by bilateral and mostly symmetrical hyperintensity on T2-weighted or FLAIR sequences, are common in older individuals.

\section{Quantitative Analysis of WMH Volume}

$\mathrm{WMH}$ volumes were quantified using FLAIR imaging by the software programs MRICRON (University of Nottingham School of Psychology, Nottingham, UK) ${ }^{2}$ and ITK-SNAP (Chen et al., 2006; Rost et al., 2010; Figure 3). All scans were checked by visual inspection. First, the MRICRON software was used to extract the effective WMH area (the first step was to import original FLAIR image, the second step was to extract the high-signal area, the third step was to sketch the contours of the affected WMH area, and the fourth step was to reconstruct the shape of WMH). Ultimately, the ITK-SNAP software was used to calculate the WMH volume.

CT and MRI scans were reviewed independently by two investigators. The test-retest intraclass correlation coefficients for inter- and intra-observer agreements were $>0.90$.

\section{Statistical Analysis}

Mean (SD) or median (interquartile range) values were used to describe continuous variables, while frequencies and percentages were used for categorical variables. Normality was determined by the Kolmogorov-Smirnov test. To compare the categorical variables of clinical features, chi-square tests were used, and the independent $t$-test was used to compare continuous variables with normal distribution while the Mann-Whitney $U$-test was used to compare continuous variables with non-normal distribution. The annual progression rate of $\mathrm{WMH}$ was calculated by subtracting the baseline $\mathrm{WMH}$ volume from the follow-up WMH volume on MRI scans, dividing this value by the baseline $\mathrm{WMH}$ volume, and finally dividing the result by year. Univariate linear regression analysis was carried out to identify potential risk factors for the annual progression rate of $\mathrm{WMH}$. Univariate predictors at a level of $p<0.10$ were considered significant and were entered

\footnotetext{
2 www mricro com
}

into a multivariate linear regression model. To determine the effect of $\mathrm{ICH}$ volume on the annual progression rate of WMH, ICH patients were categorized into quartiles according to the baseline ICH volume measured on CT. Intergroup differences were analyzed by one-way ANOVA followed by Bonferroni post hoc test. All tests were twotailed; $p<0.05$ was considered statistically significant. All statistical analyses were performed using Statistical Product and Service Solutions (SPSS) version 16.0 (SPSS Inc., Chicago, IL, USA).

\section{TABLE 1 | Baseline characteristics}

\begin{tabular}{|c|c|c|c|}
\hline Item & $\mathrm{ICH}(n=90)$ & Control $(n=90)$ & $P$ value \\
\hline Age, years, mean $\pm \mathrm{SD}$ & $66.68 \pm 13.38$ & $69.56 \pm 10.62$ & 0.11 \\
\hline Men, $n(\%)$ & 59 (66\%) & 57 (63\%) & 0.76 \\
\hline \multicolumn{4}{|l|}{ Past medical history, $n(\%)$} \\
\hline Hypertension & $48(53 \%)$ & $50(56 \%)$ & 0.09 \\
\hline Coronary artery disease & $17(19 \%)$ & $25(28 \%)$ & 0.09 \\
\hline Diabetes mellitus & $14(16 \%)$ & 27 (30\%) & 0.56 \\
\hline Hyperlipidemia & $2(2 \%)$ & $6(7 \%)$ & 0.27 \\
\hline TIA or storke & $19(21 \%)$ & $22(28 \%)$ & 0.70 \\
\hline Smoking, $n(\%)$ & $15(17 \%)$ & $18(20 \%)$ & 0.82 \\
\hline Alcohol, $n(\%)$ & $10(17 \%)$ & $15(17 \%)$ & 0.68 \\
\hline \multicolumn{4}{|l|}{ Medications, $n(\%)$} \\
\hline Use of antiplatelet agents & $19(21 \%)$ & $24(27 \%)$ & 0.95 \\
\hline Use of anticoagulation & $5(6 \%)$ & $4(7 \%)$ & 0.46 \\
\hline Use of statin & $13(14 \%)$ & $19(21 \%)$ & 0.69 \\
\hline \multicolumn{4}{|l|}{ Clinical variables, mean $\pm S D$} \\
\hline SBP, mm Hg & $144.37 \pm 20.38$ & $138.89 \pm 16.30$ & 0.07 \\
\hline DBP, $\mathrm{mm} \mathrm{Hg}$ & $78.28 \pm 10.52$ & $75.98 \pm 8.52$ & 0.14 \\
\hline FBG, mM & $5.80 \pm 1.38$ & $5.49 \pm 1.67$ & 0.26 \\
\hline HbAlc, \% & $6.06 \pm 0.51$ & $6.46 \pm 1.39$ & 0.77 \\
\hline $\mathrm{TC}, \mathrm{mM}$ & $4.16 \pm 1.35$ & $4.09 \pm 0.99$ & 0.74 \\
\hline $\mathrm{TG}, \mathrm{mM}$ & $1.48 \pm 0.71$ & $1.34 \pm 0.80$ & 0.21 \\
\hline LDL, mM & $2.20 \pm 0.92$ & $2.22 \pm 0.74$ & 0.90 \\
\hline HDL, mM & $1.09 \pm 0.38$ & $1.11 \pm 0.35$ & 0.76 \\
\hline ApoA I, g/L & $1.12 \pm 0.40$ & $1.13 \pm 0.35$ & 0.94 \\
\hline BUN, mM & $5.90 \pm 2.50$ & $6.23 \pm 3.18$ & 0.51 \\
\hline $\mathrm{Cr}, \mu \mathrm{M}$ & $90.57 \pm 86.73$ & $90.29 \pm 104.10$ & 0.99 \\
\hline $\mathrm{UA}, \mu \mathrm{M}$ & $311.15 \pm 109.71$ & $331.22 \pm 96.24$ & 0.26 \\
\hline CRP, mg/L & $15.96 \pm 34.78$ & $14.28 \pm 27.34$ & 0.75 \\
\hline
\end{tabular}

Note: values with normal distribution are presented as the mean \pm stand deviation $(S D)$. Abbreviations: $I C H$, intracerebral hemorrhage; TIA, transient ischemic attack; $S B P$, systolic blood pressure; DBP, diastolic blood pressure; FBG, fasting blood glucose; HbAlc, glycosylated hemoglobin; TC, total cholesterol; TG, triglycerides; HDL, high-density lipoprotein; $L D L$, low-density lipoprotein; ApoA I, Apolipoprotein A-l; CRP, C-reactive protein; BUN, blood urea nitrogen; Cr, creatinine; UA, uric acid. 


\section{RESULTS}

\section{Baseline Characteristics}

As shown in Table 1, differences in the demographic characteristics between the ICH and control group did not reach statistical significance $(p>0.05)$. Moreover, there were no significant differences in past medical history, medications, smoking habit, alcohol intake, or laboratory examinations between the ICH and control groups $(p>0.05)$.

\section{Analysis of the Annual Progression Rate of WMH Between the ICH and Control Groups}

A comparison of the annual progression rates of $\mathrm{WMH}$ between the ICH and control groups is shown in Table 2. The volume of WMH increased by $20 \%$ annually in the control group; however, the annual progression rate of $\mathrm{ICH}$ patients increased by $33 \%$, which was significantly higher than that of control patients ( $\mathrm{ICH}$ vs. control: $0.33 \pm 0.41$ vs. $0.20 \pm 0.32$, $p=0.01)$.

\section{Univariate and Multivariate Linear Regression of Risk Factors for the Annual Progression Rate of WMH}

We next determined the association between the annual progression rate of $\mathrm{WMH}$ and risk factors using linear regression analysis. The univariate linear regression analysis showed that ICH, hypertension history, TIA or stroke history, SBP and DBP were risk factors for the annual progression rate of WMH. However, multivariate linear regression analysis showed that only ICH and TIA or stroke history were independently associated with the progression rate of $\mathrm{WMH}$ after adjusting for other confounding variables $(p<0.05$; Table 3).

TABLE 2 | Annual progression rate of WMH between $\mathrm{ICH}$ and control group.

\begin{tabular}{lccr}
\hline & ICH & Control & P value \\
\hline $\begin{array}{l}\text { Annual progression rate } \\
\text { of } \mathrm{WMH}, \% \text {, mean }+\mathrm{SD}\end{array}$ & $0.33 \pm 0.41$ & $0.20 \pm 0.32$ & $0.01^{*}$
\end{tabular}

Note: *ICH vs. control significant difference $(P<0.05)$. Abbreviations: $I C H$, intracerebral hemorrhage; WMH, white matter hyperintensity.

TABLE 3 | Univariate and multivariate linear regression of risk factors of annual progression rate of $\mathrm{WMH}$.

\begin{tabular}{lrrrrrr}
\hline & \multicolumn{2}{c}{ Univariate } & & \multicolumn{2}{c}{ Multivariate } \\
\cline { 2 - 3 } \cline { 6 - 7 } & $\boldsymbol{\beta}$ & $\boldsymbol{P}$ value & & $\boldsymbol{\beta}$ & $\boldsymbol{P}$ value \\
\hline ICH & -0.14 & $0.01^{*}$ & & -0.17 & $0.02^{*}$ \\
Hypertension history & 0.14 & $0.03^{*}$ & & - & $-^{\#}$ \\
TIA or stroke history & -0.12 & 0.08 & & 0.14 & $0.04^{*}$ \\
SBP & 0.00 & $0.03^{*}$ & & 0.00 & 0.34 \\
DBP & 0.01 & $0.02^{*}$ & & 0.01 & 0.14
\end{tabular}

Note: * significant difference $(P<0.05)$, \# since the history of hypertension, systolic and diastolic pressure was not independent, in the multiple regression model, only SBP and $D B P$ were included. Abbreviations: $I C H$, intracerebral hemorrhage; $W M H$, white matter hyperintensity; TIA, transient ischemic attack; SBP, systolic blood pressure; DBP, diastolic blood pressure.
TABLE 4 | Univariate linear regression of $\mathrm{ICH}$ volume and $\mathrm{ICH}$ position of annual progression rate of $\mathrm{WMH}$.

\begin{tabular}{lcc}
\hline & $\boldsymbol{\beta}$ & $\boldsymbol{P}$ value \\
\hline $\mathrm{ICH}$ volume & $8.987 \mathrm{E}-6$ & $0.03^{*}$ \\
$\mathrm{ICH}$ position & 0.08 & 0.20 \\
\hline
\end{tabular}

Note: *significant difference $(P<0.05)$. Abbreviations: $I C H$, intracerebral hemorrhage; WMH, white matter hyperintensity.

\section{Linear Regression of the Association of $\mathrm{ICH}$ Volume and ICH Position With the Annual Progression Rate of WMH}

In univariate linear regression analysis, increasing ICH volume was a positive predictor of the annual progression rate of $\mathrm{WMH}$ $(p<0.05)$; specifically, a higher annual progression rate of WMH was associated with a larger ICH volume at baseline $(p=0.03)$. Nevertheless, no trend toward an association between ICH position and the annual progression rate of WMH was observed ( $p=0.20$; Table 4$)$.

\section{Analysis of the Relationship of ICH Volume and the Annual Progression Rate of WMH According to Quartiles of ICH Volume}

In addition, we further assessed the link between the effect of ICH volume and the annual progression rate of WMH. We categorized patients into four groups according to quartiles of ICH volume: group $1(0 \%-25 \%:<10 \mathrm{~mL})$, group $2(26 \%-50 \%$ : 10-20 mL), group 3 (51\%-75\%: $21-30 \mathrm{~mL}$ ) and group 4 (76\%-100\%: > $30 \mathrm{~mL})$. We found that patients in group 4 showed a $76 \%$ annually progressive rate, which is significantly higher than that in group 1 (group1 vs. group 4: $0.26 \pm 0.37$ vs. $0.76 \pm 0.45, p=0.03$; Table 5, Figure 4).

\section{DISCUSSION}

The main findings of this study were as follows: (1) ICH patients had more severe WMH progression after ICH than patients without $\mathrm{ICH}$, according to quantitative $\mathrm{WMH}$ measurements; and (2) WMH progression was associated with the ICH volume at baseline, larger ICH volume and higher annual progression rate of WMH.

It is generally known that $\mathrm{WMH}$ is a highly prevalent disease in older individuals. The presence and severity of WMH may be linked to the cumulative effects of multiple factors such as age, hypertension, cerebral infarction, lacunar infarction, history of ICH and increased TG (Zhang and Kang, 2013; Xu et al., 2014; Bivard et al., 2016). Consistent with previous studies, we also found that ICH, TIA or stroke history, SBP and DBP were associated with the progression of $\mathrm{WMH}$ in our study population. Among these risk factors, a history of ICH was recognized as the most important vascular contributor because it was an independent predictor of the annual progression rate of WMH. ICH and LA share several risk factors in common (hypertension, cerebrovascular disease), and may share a common underlying pathological mechanism involving microangiopathy (Caprio et al., 2013). Interestingly, the relationship between $\mathrm{ICH}$ and $\mathrm{WMH}$ progression is a 
TABLE 5 | Analysis of relationship of $\mathrm{ICH}$ Volume and annual progression rate of WMH according to quartered ICH Volume.

\begin{tabular}{|c|c|c|c|c|c|}
\hline & \multicolumn{5}{|c|}{ Hemorrhage volume } \\
\hline & 1 & 2 & 3 & 4 & $P$ \\
\hline & $0-25 \%(n=57)$ & $26-50 \%(n=17)$ & $51-75 \%(n=10)$ & $76-100 \%(n=6)$ & value \\
\hline Hemorrhage volume $\left(\mathrm{mm}^{3}\right)$ & $3881.93 \pm 2498.65$ & $13498.71 \pm 2739.28$ & $24795.00 \pm 3161.74$ & $36506.67 \pm 4249.57$ & - \\
\hline Annual progression rate of WMH (\%) & $0.26 \pm 0.37$ & $0.40 \pm 0.29$ & $0.37 \pm 0.71$ & $0.76 \pm 0.45$ & $0.02^{*}$ \\
\hline
\end{tabular}

Note: values are presented as the mean $\pm S D$; *intergroup differences $(P=0.02), 1$ vs. 4 significant difference $(P=0.03)$. Abbreviations: ICH, intracerebral hemorrhage; $W M H$, white matter hyperintensity.

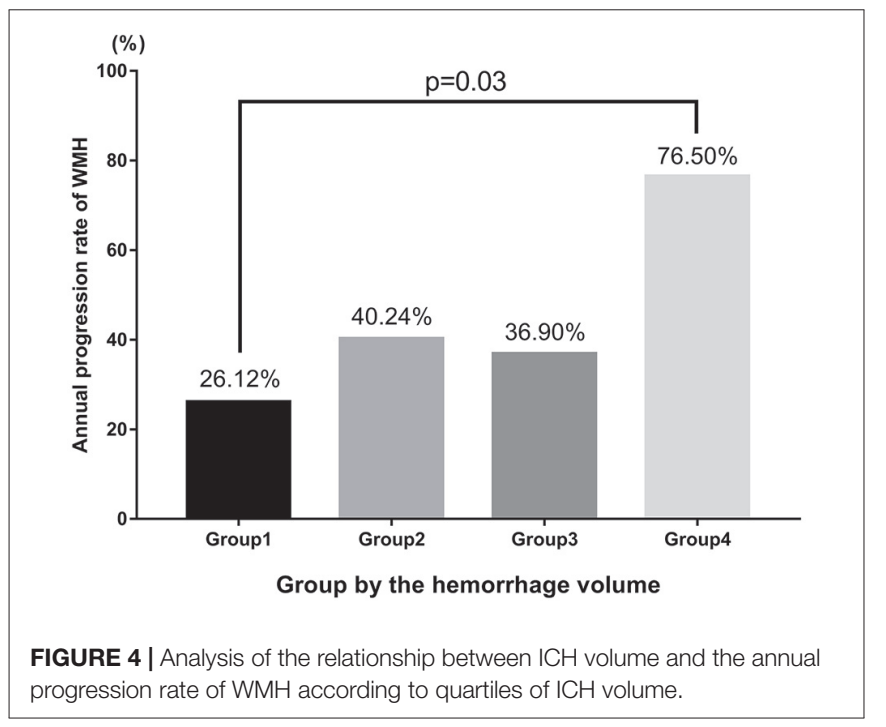

mutually promoting process, not merely a unilateral process. Recent studies have shown that severe WMH was associated with spontaneous lobar hemorrhage and a greater risk of local hemorrhage after intravenous thrombolysis (IVT; Chen et al., 2018). In addition, more extensive WM damage was detected in patients with ICH than in those with ischemic stroke or other cerebral small vessel pathologies (Rost et al., 2010).

Most single hemorrhages were found in deep (subcortical) sites, including the basal ganglia (34.2\%), thalamus (8.3\%), cerebellum (6.8\%), ventricles $(1.5 \%)$ and brainstem $(1.1 \%$; $\mathrm{Hu}$ et al., 2013). However, we do not know whether the ICH position affects the progression rate of $\mathrm{WMH}$. One previous publication indicated that the WMH volume was similar in deep hemispheric ICH and lobar ICH and did not find major differences in supratentorial WMH distribution (Smith et al., 2017). In this study, we also did not find a significant association between the annual progression rate of $\mathrm{WMH}$ and $\mathrm{ICH}$ position, which was consistent with the results of Smith et al. (2010). Increasing research on WMI after hypertensive ICH should focus on these sites in the future.

Compared to ischemic stroke, ICH has higher mortality and leads to more severe disability (Keep et al., 2012). Known poor prognostic factors of ICH include large hematoma volume, hematoma expansion, intraventricular hemorrhage, infratentorial location, older age and anticoagulation treatment (An et al., 2017); hence, ICH volume is an important factor in determining the prognosis of $\mathrm{ICH}$ patients. A previous study found that a hematoma volume exceeding approximately $25 \mathrm{~mL}$ was a precondition for the occurrence of WM damage (Sugimoto et al., 1989). A clear link between the progression of $\mathrm{WMH}$ and volume of ICH was observed in our study. The progression of $\mathrm{WMH}$ was volume-dependent, and the group with the highest ICH volume had a significantly higher annual progression rate of WMH. Massive ICH volume can trigger more serious WM damage.

ICH is usually caused by ruptured vessels that are degenerated due to long-standing hypertension. Cerebral autoregulation (CA) is a mechanism which protects brain tissue from either hyperperfusion or hypoperfusion within a wide range of BP fluctuations (Ma et al., 2016). Dysfunction of automatic regulation could be the prime contributor to the occurrence of $\mathrm{ICH}$. Notably, WMH is also attributed to impairment of cerebral hemeodynamics, because the development and progression of $\mathrm{WMH}$ occur, in part, as a result of regional hypoperfusion of susceptible normalappearing WM (Etherton et al., 2016). Therefore, WMH and ICH can have a common pathological pathway (Zuo et al., 2017). Furthermore, ICH could exacerbate demyelination and downregulation of myelin basic protein (MBP) expression in the WM. Many pathological molecules such as TNF$\alpha$ and cytokines were highly expressed or overactivated. These pathological molecules had aggravated the damage to myelin sheaths and then affected the morphology and function of the WM (Zuo et al., 2017). Increasing research has suggested that WMI will seriously influence the prognosis of hypertensive ICH patients (Callisaya et al., 2015). Severe WMI may reflect the vulnerability of the brain to further insults and predicts poor outcome after ICH (Lee et al., 2010; Tveiten et al., 2013; Lambert et al., 2016; Yamashita et al., 2016). In summary, WMI after hypertensive ICH was caused by a variety of pathogenic factors and resulted in a worse long-term prognosis. Further research is needed to explore the exact pathogenic mechanisms for WMI after hypertensive ICH.

However, this study has some limitations. First, although the records of quantities of patients were screened, only 90 patients met the inclusion criteria, which decreased the statistical power of this study. Second, although the interval between two MRI examinations is usually around 1 year, the accuracy of the annual progression rate of $\mathrm{WMH}$ may be influenced by the large range of follow up time ( 3 months to 3 years). Finally, $\mathrm{WMH}$ is a reaction to a serious WMI; thus, we may ignore the disruption of normalappearing WM. Recently, diffusion tensor imaging (DTI) was shown to be the most suitable for detecting microstructural abnormalities in WM at the early stages (Jang et al., 2013; Goh et al., 2015). To better understand the damage to WM fibers, 
research using DTI should be the top priority in the future (Tamura and Araki, 2015).

\section{CONCLUSION}

In conclusion, our study was the first to use quantitative analysis to show that $\mathrm{ICH}$ could trigger more severe $\mathrm{WMH}$, and that a larger ICH volume was associated with a greater progression of WMH after ICH. Consequently, our findings suggested that the progression of WMH might provide important prognostic information about patients with $\mathrm{ICH}$ and could be proposed as a clinically relevant disease marker. Thus, it is important to control the risk factors to reduce the occurrence of $\mathrm{ICH}$ and improve the functional outcomes of patients after ICH. Additionally, the study confirms the importance of pharmacologically modulating $\mathrm{WMH}$ volume at the early stages of WMH development. Further

\section{REFERENCES}

An, S. J., Kim, T. J., and Yoon, B. W. (2017). Epidemiology, risk factors, and clinical features of intracerebral hemorrhage: an update. J. Stroke 19, 3-10. doi: 10.5853/jos.2016.00864

Bivard, A., Cheng, X., Lin, L. T., Levi, C., Spratt, N., Kleinig, T., et al. (2016). Global white matter hypoperfusion on CT predicts larger infarcts and hemorrhagic transformation after acute ischemia. CNS Neurosci. Ther. 22, 238-243. doi: $10.1111 / \mathrm{cns} .12491$

Callisaya, M. L., Beare, R., Phan, T., Blizzard, L., Thrift, A. G., Chen, J., et al. (2015). Progression of white matter hyperintensities of presumed vascular origin increases the risk of falls in older people. J. Gerontol. A Biol. Sci. Med. Sci. 70, 360-366. doi: 10.1093/gerona/glu148

Caprio, F. Z., Maas, M. B., Rosenberg, N. F., Kosteva, A. R., Bernstein, R. A., Alberts, M. J., et al. (2013). Leukoaraiosis on magnetic resonance imaging correlates with worse outcomes after spontaneous intracerebral hemorrhage. Stroke 44, 642-646. doi: 10.1161/strokeaha.112.676890

Chen, Y. W., Gurol, M. E., Rosand, J., Viswanathan, A., Rakich, S. M., Groover, T. R., et al. (2006). Progression of white matter lesions and hemorrhages in cerebral amyloid angiopathy. Neurology 67, 83-87. doi: 10.1212/01.wnl.0000223613.57229.24

Chen, Y., Yan, S., Xu, M., Zhong, G., Liebeskind, D. S., and Lou, M. (2018). More extensive white matter hyperintensity is linked with higher risk of remote intracerebral hemorrhage after intravenous thrombolysis. Eur. J. Neurol. 25:380-e15. doi: 10.1111/ene.13517

de Leeuw, F.-E., de Groot, J. C., Achten, E., Oudkerk, M., Ramos, L. M., Heijboer, R., et al. (2001). Prevalence of cerebral white matter lesions in elderly people: a population based magnetic resonance imaging study. The Rotterdam scan study. J. Neurol. Neurosurg. Psychiatry 70, 9-14. doi: 10.1136/jnnp.70.1.9

Etherton, M. R., Wu, O., and Rost, N. S. (2016). Recent advances in leukoaraiosis: white matter structural integrity and functional outcomes after acute ischemic stroke. Curr. Cardiol. Rep. 18:123. doi: 10.1007/s11886-016-0803-0

Goh, S. Y., Irimia, A., Torgerson, C. M., Tubi, M. A., Real, C. R., Hanley, D. F., et al. (2015). Longitudinal quantification and visualization of intracerebral haemorrhage using multimodal magnetic resonance and diffusion tensor imaging. Brain Inj. 29, 438-445. doi: 10.3109/02699052.2014.989907

Hu, Y. Z., Wang, J. W., and Luo, B. Y. (2013). Epidemiological and clinical characteristics of 266 cases of intracerebral hemorrhage in Hangzhou, China. J. Zhejiang Univ. Sci. B 14, 496-504. doi: 10.1631/jzus.b1200332

Jang, S. H., Choi, B. Y., Chang, C. H., Jung, Y. J., Byun, W. M., Kim, S. H., et al. (2013). The effects of hydrocephalus on the periventricular white matter in intracerebral hemorrhage: a diffuser tensor imaging study. Int. J. Neurosci. 123, 420-424. doi: 10.3109/00207454.2012.763164

Keep, R. F., Hua, Y., and Xi, G. (2012). Intracerebral haemorrhage: mechanisms of injury and therapeutic targets. Lancet Neurol. 11, 720-731. doi: 10.1016/s14744422(12)70104-7

Lambert, C., Benjamin, P., Zeestraten, E., Lawrence, A. J., Barrick, T. R., and Markus, H. S. (2016). Longitudinal patterns of leukoaraiosis and studies should be undertaken to elucidate the pathophysiologic link between WMH and ICH and to confirm our findings.

\section{AUTHOR CONTRIBUTIONS}

All authors had full access to all the data in the study and take responsibility of the data and the accuracy of the data analysis. XuemeiC: study concept and design. XuemeiC, XinC and MX: acquisition of data. YC and XuemeiC: statistical analysis. XuemeiC and XinC: visualization. XuemeiC, XinC, YC, MX, TY and JL: writing — review and editing.

\section{FUNDING}

This research was supported by the Nanjing Medical Science and Technique Development Foundation (QRX17032).

brain atrophy in symptomatic small vessel disease. Brain 139, 1136-1151. doi: 10.1093/brain/aww009

Lee, S. H., Kim, B. J., Ryu, W. S., Kim, C. K., Kim, N., Park, B. J., et al. (2010). White matter lesions and poor outcome after intracerebral hemorrhage: a nationwide cohort study. Neurology 74, 1502-1510. doi: 10.1212/wnl.0b013e3181d d425a

Lou, M., Al-Hazzani, A., Goddeau, R. P. Jr., Novak, V., and Selim, M. (2010). Relationship between white-matter hyperintensities and hematoma volume and growth in patients with intracerebral hemorrhage. Stroke 41, 34-40. doi: 10.1161/strokeaha.109.564955

Ma, H., Guo, Z. N., Liu, J., Xing, Y., Zhao, R., and Yang, Y. (2016). Temporal course of dynamic cerebral autoregulation in patients with intracerebral hemorrhage. Stroke 47, 674-681. doi: 10.1161/strokeaha.115.011453

Rost, N. S., Rahman, R. M., Biffi, A., Smith, E. E., Kanakis, A., Fitzpatrick, K., et al. (2010). White matter hyperintensity volume is increased in small vessel stroke subtypes. Neurology 75, 1670-1677. doi: 10.1212/wnl.0b013e3181f c279a

Smith, E. E., Nandigam, K. R., Chen, Y. W., Jeng, J., Salat, D., Halpin, A., et al. (2010). MRI markers of small vessel disease in lobar and deep hemispheric intracerebral hemorrhage. Stroke 41, 1933-1938. doi: 10.1161/strokeaha.110. 579078

Smith, E. E., Saposnik, G., Biessels, G. J., Doubal, F. N., Fornage, M., Gorelick, P. B., et al. (2017). Prevention of stroke in patients with silent cerebrovascular disease: a scientific statement for healthcare professionals from the american heart association/american stroke association. Stroke 48, e44-e71. doi: 10.1161/str. 0000000000000116

Srikanth, V., Phan, T. G., Chen, J., Beare, R., Stapleton, J. M., and Reutens, D. C. (2010). The location of white matter lesions and gait-a voxel-based study. Ann. Neurol. 67, 265-269. doi: 10.1002/ana.21826

Sugimoto, K., Minematsu, K., and Yamaguchi, T. (1989). [Aphasia and size of hematoma in patients with left putaminal hemorrhage]. Rinsho Shinkeigaku 29, 574-578.

Tamura, Y., and Araki, A. (2015). Diabetes mellitus and white matter hyperintensity. Geriatr. Gerontol. Int. 15, 34-42. doi: 10.1111/ggi.12666

Tveiten, A., Ljostad, U., Mygland, A., and Naess, H. (2013). Leukoaraiosis is associated with short- and long-term mortality in patients with intracerebral hemorrhage. J. Stroke Cerebrovasc. Dis. 22, 919-925. doi: 10.1016/j. jstrokecerebrovasdis.2013.01.017

Wardlaw, J. M., Smith, E. E., Biessels, G. J., Cordonnier, C., Fazekas, F., Frayne, R., et al. (2013). Neuroimaging standards for research into small vessel disease and its contribution to ageing and neurodegeneration. Lancet Neurol. 12, 822-838. doi: 10.1016/S1474-4422(13)70124-8

Wen, A. Y., Wu, B. T., Xu, X. B., and Liu, S. Q. (2018). Clinical study on the early application and ideal dosage of urokinase after surgery for hypertensive intracerebral hemorrhage. Eur. Rev. Med. Pharmacol. Sci. 22, 4663-4668. doi: 10.26355/eurrev_201807_15526

Xu, W. H., Li, M. L., Niu, J. W., Feng, F., Jin, Z. Y., and Gao, S. (2014). Intracranial artery atherosclerosis and lumen dilation in cerebral small-vessel diseases: a 
high-resolution MRI study. CNS Neurosci. Ther. 20, 364-367. doi: 10.1111/cns. 12224

Yamashita, Y., Wada, I., Horiba, M., and Sahashi, K. (2016). Influence of cerebral white matter lesions on the activities of daily living of older patients with mild stroke. Geriatr. Gerontol. Int. 16, 942-947. doi: 10.1111/ggi.12580

Zhang, S., and Kang, X. (2013). Investigation of the risk factors for leukoaraiosis (LA). Asia Pac. J. Public Health 25, 64S-71S. doi: 10.1177/10105395134 93111

Zuo, S., Pan, P., Li, Q., and Chen, Y. (2017). White matter injury and recovery after hypertensive intracerebral hemorrhage. Biomed Res. Int. 2017:6138424. doi: $10.1155 / 2017 / 6138424$
Conflict of Interest Statement: The authors declare that the research was conducted in the absence of any commercial or financial relationships that could be construed as a potential conflict of interest.

Copyright (c) 2018 Chen, Chen, Chen, Xu, Yu and Li. This is an open-access article distributed under the terms of the Creative Commons Attribution License (CC BY). The use, distribution or reproduction in other forums is permitted, provided the original author(s) and the copyright owner(s) are credited and that the original publication in this journal is cited, in accordance with accepted academic practice. No use, distribution or reproduction is permitted which does not comply with these terms. 